\title{
Determinación de las características mecánicas de los muros de fábrica de ladrillo en la arquitectura doméstica sevillana de los siglos XVIII Y XIX
}

\author{
Determination of the mechanical characteristics of \\ masonry walls of the traditional housing in Sevilla between \\ 1700 and 1900 \\ F. Pérez-Gálvez ${ }^{(*)}$, C. Rodríguez-Liñán ${ }^{(*)}$, P. Rubio ${ }^{(*)}$
}

RESUMEN

El resultado del trabajo permite manejar datos geométricos, constructivos y mecánicos objetivos en el momento de la recuperación de los muros estudiados y los edificios referidos. Las fases seguidas para el desarrollo del método han sido:

\footnotetext{
- Análisis de edificios objeto de rehabilitación: características constructivas del muro a estudiar, levantamiento de lesiones.

- Extracción y análisis en laboratorio de pequeñas muestras de la fábrica para la determinación de las características resistentes de sus materiales.

- Trabajo experimental en laboratorio para elaboración de un modelo que permita la obtención de la resistencia a compresión de la fábrica estudiada.

- Aplicación del acelerógrafo para medir la rigidez del edificio in situ, aproximándonos al módulo de elasticidad del muro de fábrica.

- Obtención de conclusiones con la comparación de valores máximos de tensiones obtenidos para una fábrica no fisurada mediante un Análisis de Elementos Finitos, con los valores obtenidos de resistencia en los estudios anteriores. Esta comparación permitirá decidir el comportamiento de los muros ante la restauración.
}

876-12

Palabras clave: vivienda, fábrica de ladrillo, restauración, parámetros resistentes.

\section{SUMMARY}

The main aim consists on improving the information technology tools in order to make models of the real situation (constructive, geometrical, resistant) of the structural elements, as well as getting the restoration or support proposals. We have developed the work in some stages:

- Analysis, inspection, measurement, and evolution of the building.

- Extraction and analysis in laboratory of small samples of masonry walls to determine the strength parameters of materials.

- Experimental studie for determination of a model and obtaining the compression stresses of the masonry walls studied.

- The monitoring instrumentation which has been used is an accelerograph to evaluate the rigidity of the walls.

- Application of Finite Elements Method in order to obtain the stresses distribution before and after any change in the building. The final aim is to be able to compare the results from the laboratory and the stresses distribution from the F.E.M., in order to decide the best way of getting the restoration.

Keywords: traditional housing, masonry walls, restoration, strength parameters. 


\section{DEFINICIÓN DEL PROBLEMA Y OBJE- TIVOS PROPUESTOS}

En el campo de la Arquitectura es necesario actuar con rigor y conocimiento exhaustivo de los problemas cuando se trata de valorar elementos estructurales. Esto cobra especial relevancia en proyectos de rehabilitación donde el desconocimiento del estado actual de los elementos resistentes repercute en el desarrollo del proyecto arquitectónico, así como en el proceso de ejecución. En la ciudad de Sevilla este problema se ve incrementado debido a la extensión de su Casco Histórico, así como por la Normativa Especial de Protección que se está promoviendo por parte de las instituciones implicadas.

El interés por proteger y mantener nuestro legado histórico, afecta no sólo a grandes edificios de carácter monumental sino a la mayoría de pequeños y modestos edificios que configuran lo que hoy llamamos centro histórico de la ciudad, sin embargo se margina a la arquitectura popular en investigaciones históricas, constructivas y estructurales.

El estudio y la constante recuperación del tejido residencial formado por una arquitectura doméstica y humilde es la mejor garantía de la conservación de la ciudad histórica.

Por otra parte, los materiales actuales cuentan con un proceso de fabricación y control que garantizan los valores de cálculo que utilizamos; los materiales antiguos y concretamente los que forman las obras de fábrica dependen de muchos factores ajenos al proceso de fabricación a la hora de determinar sus parámetros resistentes: edad, proceso de deterioro, estado de conservación, evolución del estado de cargas. Es importante determinar la capacidad portante de los elementos de obra de fábrica de las edificaciones históricas a partir de datos que tengan en cuenta esas variables.

La determinación de un método de cálculo que aporte todos estos valores nos ha llevado al desarrollo de una investigación cuyos objetivos son:

1. Desarrollar de una metodología no destructiva y rigurosa basada en ensayos mecánicos, dinámicos y químicos, y en métodos de cálculo mediante elementos finitos (análisis elástico lineal), para la determinación del estado de tensiones de muros de obras de fábrica pertenecientes a la Arquitectura Doméstica Sevillana de los siglos XVIII-XIX.

2. Obtener un valor de Resistencia a Compresión, módulo de deformación y estado tensional de la fábrica para distintas hipótesis de carga.
3. Conseguir a partir del método propuesto un criterio objetivo del estado y comportamiento de los muros para una posible intervención.

En definitiva, el método propuesto proporcionará datos sobre los parámetros resistentes de la fábrica, permitiendo al arquitecto manejar resistencias reales de cálculo y valorar el estado actual de las fábricas con un criterio más objetivo que el de la inspección visual o el valor histórico de un edificio.

\subsection{Interés del estudio y límites del tra- bajo}

Los límites de actuación se establecen en la ciudad de Sevilla, por la extensión de edificios protegidos por la normativa en vigor y la prolífica actuación profesional en este campo. Para valorar el interés de la investigación se realizó una minuciosa labor de análisis del estado actual del caserío en el centro histórico de Sevilla.

Se han estudiado en los distintos Planes Especiales de Protección actuales la situación de los edificios que conforman la mayor parte de la trama urbana histórica (edificios residenciales de los siglos XVIII- XIX) en los que se obliga al mantenimientos de los muros portantes de fachada en todos los casos (calificados como niveles $C, D, y$ E). El resultado ha sido que un porcentaje aproximado del $47 \%$ de los edificios afectados están o estarán a corto o medio plazo en proceso de rehabilitación que requerirá un estudio de estabilidad de los muros de fábrica de ladrillo que forman las fachadas.

En Sevilla el problema parece de gran trascendencia para los técnicos que se enfrentan a diario con la rehabilitación de esta Arquitectura popular y que deben garantizar con su intervención el mantenimiento de tipologías y muros de fachada con las actuales exigencias de seguridad de la normativa actual.

En este ámbito de trabajo podrán incluirse igualmente aquellas ciudades en las que exista una arquitectura similar a la estudiada. Los límites de la investigación están en el estudio de fachadas formadas por muros portantes de fábrica de ladrillo y mortero de cal con una tipología definida y unas características constructivas concretas.

Se determinará su capacidad portante a partir de un análisis objetivo de los materiales que lo componen, por lo que los resultados varían en función del estado actual del elemento. Es necesario pues el estudio individualizado de cada elemento independientemente que pertenezca al ámbito de trabajo definido. Se desarrolla un método para verificar la estabilidad estructural de los elementos 
estudiados con estudios comparativos entre estado de los materiales, estado actual del elemento ante acciones dinámicas y estados tensionales para distintas hipótesis de carga. Se pretende con ello saber las tensiones máximas que permiten los muros, partiendo de una fábrica sana y comparando los valores de los mapas de tensiones obtenidos por el M.E.F. Al trabajar en régimen elástico lineal, sabremos las zonas inicialmente más tensionadas y obtener la tensión de rotura, pero no permite el estudio de redistribuciones debido a la aparición de fisuras y grietas. En todos los casos estudiados se han podido verificar las zonas de máximas tensiones con las zonas fisuradas que indican el agotamiento de la fábrica. En ningún caso se trata de conocer la evaluación de la fábrica fisurada, ni el estudio de redistribuciones tensionales por la aparición de grietas y fisuras, que requeriría un análisis no lineal, discutible y cuestionable en una fábrica con grandes heterogeneidades como la que tratamos.

\section{ANTECEDENTES}

Partimos de edificios con una tipología formal y constructiva común que ha sido necesario analizar para valorar la uniformidad de la arquitectura objeto de nuestro estudio. La organización formal de la Arquitectura doméstica sevillana de los siglos XVIII y XIX ha sido estudiada profundamente por otros autores (2), pero podemos concluir en sus características formales comunes:

- Fachadas. Limitadas por el zócalo en la parte inferior y cornisa en la superior. Bandas horizontales poco labradas Ilamadas impostas marcan las distintas alturas de la primera crujía (dos o tres plantas). Los huecos suelen ser rectangulares enmarcados con sencillos marcos en otro color (Fig. 1).

- Cubiertas. Dos tipos: azotea y tejado, dependiendo de la organización de la recogida de aguas, siendo ésta hacia el exterior en la primera crujía. Las cubiertas reflejan los distintos muros de carga que definen el sistema estructural del edificio.

- Patio: Es el elemento característico y fundamental de la casa sevillana, por la irregularidad y profundidad del parcelario es imprescindible. En torno a él se organizan las crujías del edificio.

Los sistemas constructivos han sido fundamentales para definir el ámbito de actuación común del trabajo. Exponemos a continuación los que definen los muros de fábrica de fachada:

- Cimentación. Consiste en la prolongación del muro de fábrica hasta un estrato firme (1- 1,5 m), en algunos casos con ensanchamiento en las últimas hiladas.

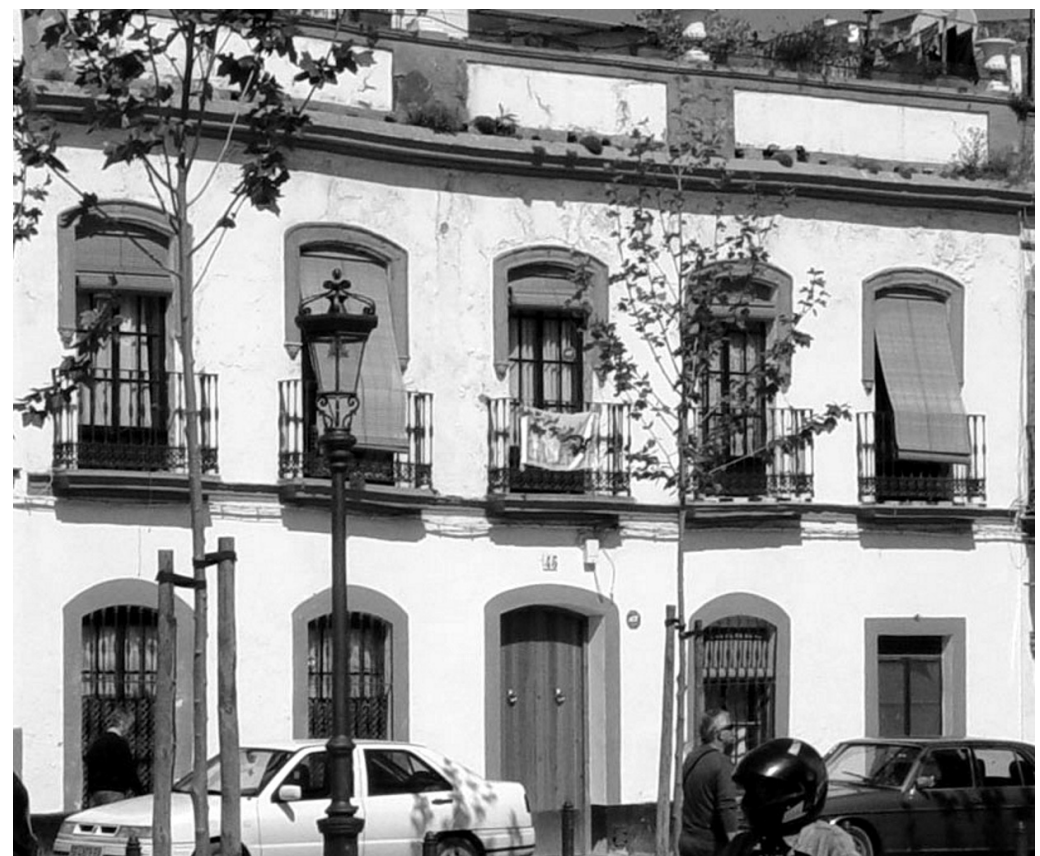

- Fábrica. Formada por ladrillos macizos de tejar y morteros de cal de dimensiones 2 pies $(50-60 \mathrm{~cm})$, con llagueado horizontal 3-5 cm y tendeles 2-4 cm. La fábrica se construye trabada pero sin regla fija en la traba ni en el aparejo (Fig. 2).

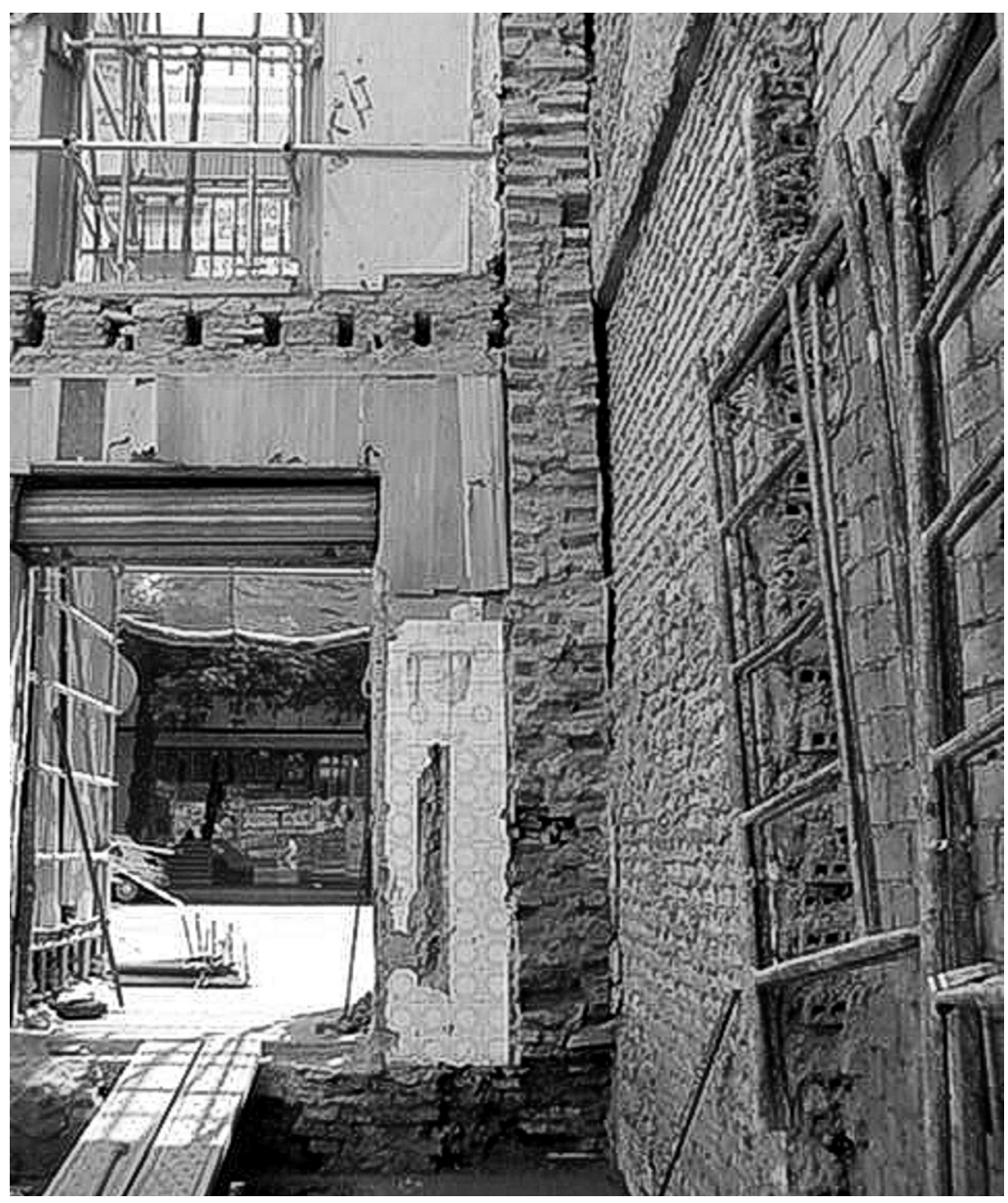


3. Sección constructiva de cornisa de Alameda de Hércules 45

4. Sección constructiva por vuelo de balcones, c/ Feria 97
- Huecos. Habitualmente se realizan con dinteles de madera en el interior y arco adintelado en el exterior (Fig. 3).

- Cornisas e impostas. Se realizan con hiladas a soga y tizón voladas hasta el máximo de 1 pie sobre el plano de fachada.

- Balcones. Variedad de soluciones, habitualmente con perfiles de hierro que se apoyan en el muro de diferente forma, sobre ellos tablazón de piezas cerámicas, relleno y solería (Fig. 4).
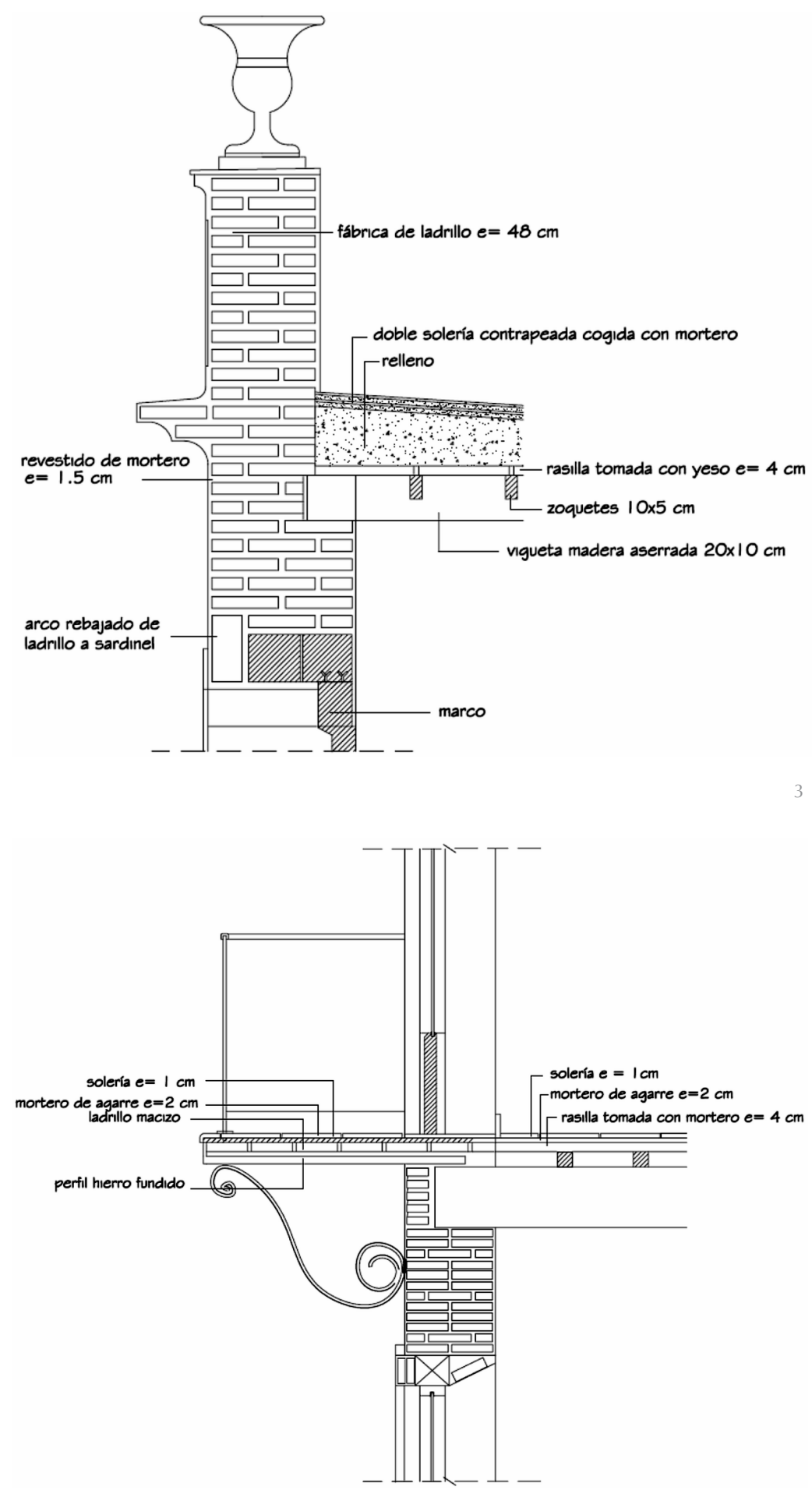

\section{MÉTODO DE ANÁLISIS}

Para poder explicar el método de forma práctica y sencilla utilizaremos uno de los edificios estudiados donde se ha aplicado el análisis propuesto en el muro de fachada.

Paso a paso seguiremos el proceso y la interpretación de los resultados en el edificio de calle Caballerizas 10 - 12 de Sevilla.

\section{Análisis Histórico.}

Fase que requerirá un estudio de la documentación histórica existente y permitirá una primera datación del edificio. En el caso de calle Caballerizas se trata de un edificio sin catalogar, cuya fachada carece de elementos singulares. Por su tipología se puede datar del siglo XIX.

Análisis del edificio.

Se requerirá un levantamientos planimétrico que incluya plantas del edificio (Fig. 5), alzados y un plano de levantamiento de daños exhaustivo del muro de fábrica a analizar (Fig.6). Se ha analizado para cada lesión las posibles causas, apareciendo lesiones debidas a dilataciones térmicas en los pretiles, agotamiento de dinteles y fisuras y grietas debidas al agotamiento de la fábrica.

Serán éstas últimas las que nos ayudarán con los ensayos complementarios a determinar la capacidad resistente de la fábrica. Igualmente el análisis constructivo de dicho muro y el levantamiento de la sección constructiva por fachada nos aportará datos sobre espesor de la fábrica, Ilagueados, formación de huecos y cornisas, forjados, cimentación y cubierta. Es esta fase la que nos permite verificar la similitud constructiva de todos los edificios estudiados pertenecientes a la misma época .

\subsection{Extracción de muestras y análisis en laboratorio}

Será preciso determinar (Tablas 1-4, pág. 24):

- Geometría de la fábrica. Medición in situ y sobre las muestras en laboratorio.

- Composición química del mortero.

- Características fisico-mecánicas del mortero y del ladrillo. Análisis granulométrico de los componentes del mortero y resistencia a compresión del mortero y el ladrillo.

En el caso que nos ocupa obtuvimos los siguientes resultados:

Geometría de la fábrica. Fábrica de ladrillo de $54 \mathrm{~cm}$ de espesor, tomados con mortero de cal. 
Se determinó la siguiente geometría:

- Ladrillo macizo artesano de $(29+/-2) \times(14+$ $/-1) \times(5+/-2) \mathrm{cm}$.

- Llagueado horizontal de $4+/-1 \mathrm{~cm}$.

- Llagueado vertical de $5+/-2 \mathrm{~cm}$.

De los resultados de los ensayos se deduce que:

- La resistencia mecánica de los ladrillos es bastante buena.

- La proporción de óxido de cal no carbonatado es muy baja, incluso con valores del $0 \%$ en alguna muestra. En condiciones normales, el óxido de cal no carbonatado en relación al dosificado inicialmente debe de estar en una proporción del 50\% para los primeros 100 años de antigüedad. Por lo tanto se puede determinar la edad del edificio siendo ésta de, al menos, 100 años.

- Los morteros de cal son ricos en conglomerantes 1:3 en origen, indican que el mortero se dosificó correctamente e incluso con contenidos en conglomerante ligeramente superior a lo normal en algunas muestras. El problema está en su alto contenido en finos, entre el $25 \%$ y el $37 \%$. Ésta es una característica común en los edificios estudiados y la principal causa del pésimo envejecimiento de los morteros.

- La resistencia admisible a compresión de la fábrica se ha podido determinar por ensayos.

La imposibilidad de extraer muestras de la fábrica para la determinación de la resistencia de la misma a causa de la disgregación de los morteros, situación repetida en todos los edificios estudiados, nos llevó a plantear un trabajo experimental para poder llegar a obtener dichas resistencias (4).

Este trabajo constituyó un largo proceso de experimentación en laboratorio hasta conseguir crear probetas con ladrillos procedentes de estas construcciones y un material que simula la resistencia a compresión de los morteros con los que estamos trabajando (Fig. 7).

La rotura a compresión de familias de dichas probetas nos ha proporcionado un dato experimental sobre la resistencia a compresión característica de las fábricas de las construcciones analizadas siendo ésta de 0,85 N/ $\mathrm{mm}^{2}$ (Fig. 8).

Este dato coincide con los valores mínimos obtenidos de la bibliografía específica y en la experiencia de otros muros de similares características en los que se ha llegado a
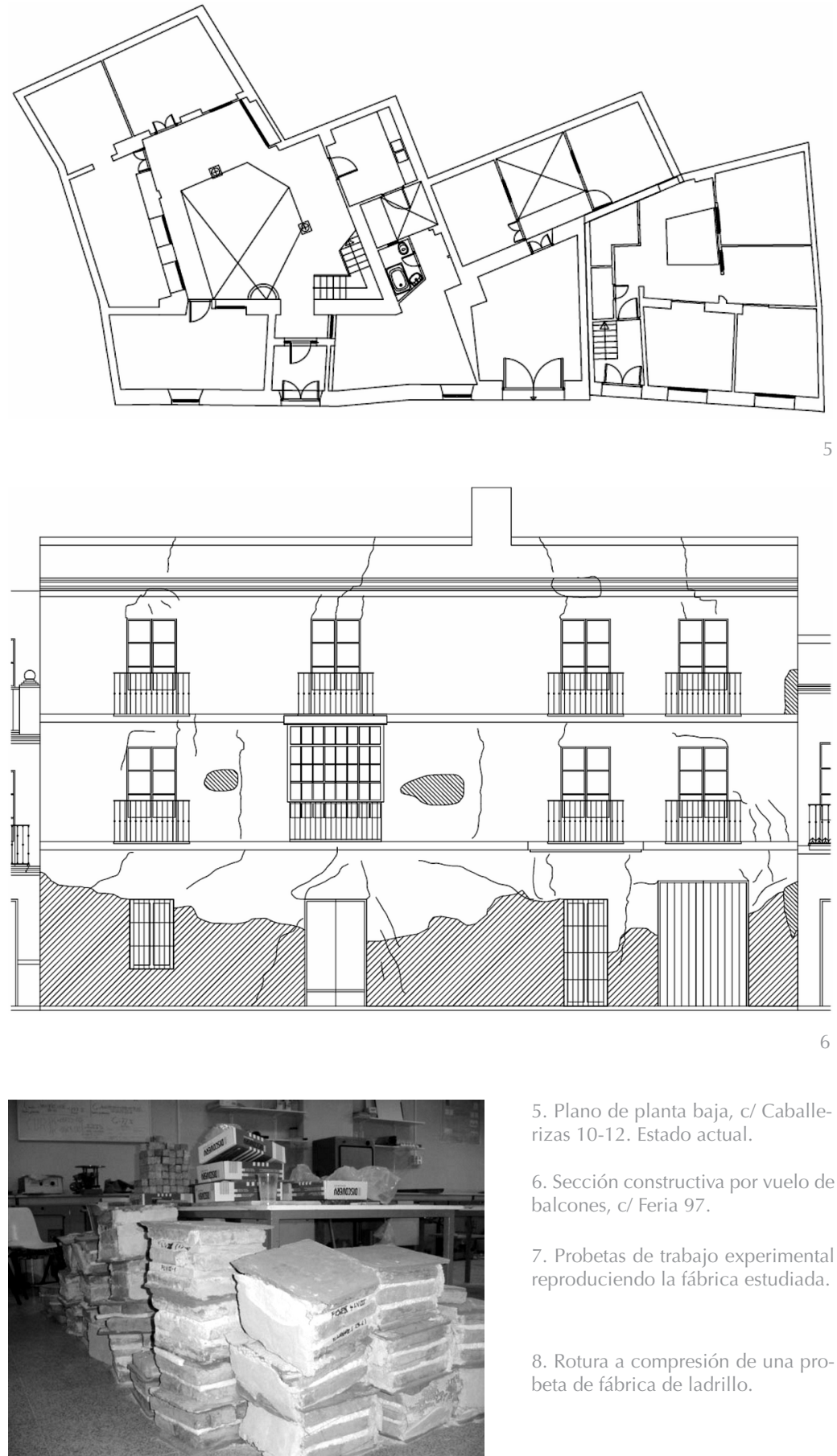

5. Plano de planta baja, c/ Caballerizas 10-12. Estado actual.

6. Sección constructiva por vuelo de balcones, c/ Feria 97

7. Probetas de trabajo experimenta reproduciendo la fábrica estudiada.

8. Rotura a compresión de una probeta de fábrica de ladrillo.

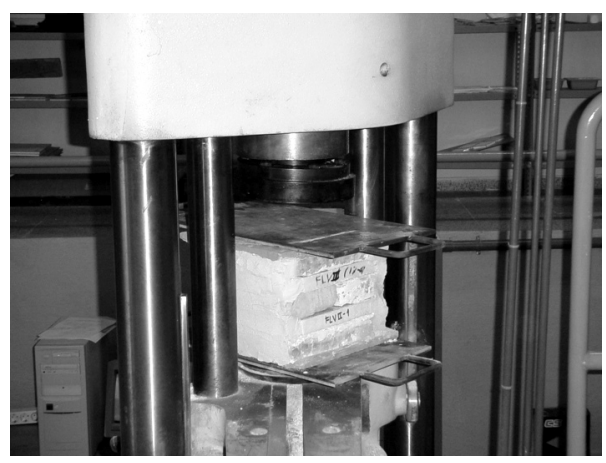


Tabla 1

Composición química del mortero

\begin{tabular}{|c|c|c|c|}
\hline $\begin{array}{c}\text { № } \\
\text { MUESTRA }\end{array}$ & $\begin{array}{c}\text { CONTENIDO EN } \\
\text { CARBONATOS } \\
\% \mathrm{CO}_{3} \mathrm{Ca}\end{array}$ & $\begin{array}{c}\text { CONTENIDO DE } \\
\text { ÓXIDO DE CALCIO } \\
\text { (cal dosificada } \\
\text { inicialmente) } \% \mathrm{CaO}\end{array}$ & $\begin{array}{c}\text { CONTENIDO DE } \\
\text { ÓXIDO DE } \\
\text { CALCIO NO } \\
\text { CARBONATADO } \\
\% \text { CaO }\end{array}$ \\
\hline $\mathrm{A}$ & 39,29 & 25,0 & 2,99 \\
\hline $\mathrm{B}$ & 41,96 & 30,5 & 7,00 \\
\hline $\mathrm{C}$ & 45,11 & 28,6 & 3,34 \\
\hline $\mathrm{D}$ & 49,20 & 32,5 & 4,95 \\
\hline $\mathrm{E}$ & 47,43 & 27,1 & 0,54 \\
\hline $\mathrm{F}$ & 35,88 & 24,6 & 4,51 \\
\hline $\mathrm{G}$ & 42,26 & 22,7 & 0,00 \\
\hline
\end{tabular}

Tabla 2

Dosificación inicial en volumen cal/áridos

\begin{tabular}{|c|c|}
\hline $\begin{array}{c}\text { № } \\
\text { MUESTRA }\end{array}$ & $\begin{array}{c}\text { DOSIFICACIÓN } \\
\text { VOLUMEN } \\
\text { CAL/ÁRIDO }\end{array}$ \\
\hline $\mathrm{A}$ & $1 / 3$ \\
\hline $\mathrm{B}$ & $1 / 2,27$ \\
\hline $\mathrm{C}$ & $1 / 2,49$ \\
\hline $\mathrm{D}$ & $1 / 2,07$ \\
\hline $\mathrm{E}$ & $1 / 2,69$ \\
\hline $\mathrm{F}$ & $1 / 3,06$ \\
\hline $\mathrm{G}$ & $1 / 3,40$ \\
\hline
\end{tabular}

es medir la rigidez estructural, con una estimación aproximada del periodo. A partir de estos datos nos aproximaremos al valor del módulo de elasticidad de la fábrica.

Para determinar el periodo es preciso determinar las frecuencias y amortiguaciones del edificio. En el caso del muro estudiado en Caballerizas los acelerógrafos fueron colocados en el hueco del balcón de la segunda planta, en dos posiciones horizontales (paralelo y perpendicular a la fachada) y vertical. De esta forma se obtuvieron tres medidas de señales para la determinación de las frecuencias de vibración. Una vez registrada la señal, y analizada en laboratorio pudieron medirse las frecuencias fundamentales mediante el analizador. El análisis dinámico nos dio una frecuencia del edificio de $0,65 \mathrm{a} 0,8 \mathrm{~Hz}$, o lo que es igual, un periodo de $1 / 0,8=1,25 \mathrm{~s}$.

\subsection{Interpretación de los resultados. Aproximación a las propiedades del material}

Para poder valorar comparativamente los datos obtenidos de los distintos ensayos sobre

Tabla 3

Características físico-mecánicas del mortero

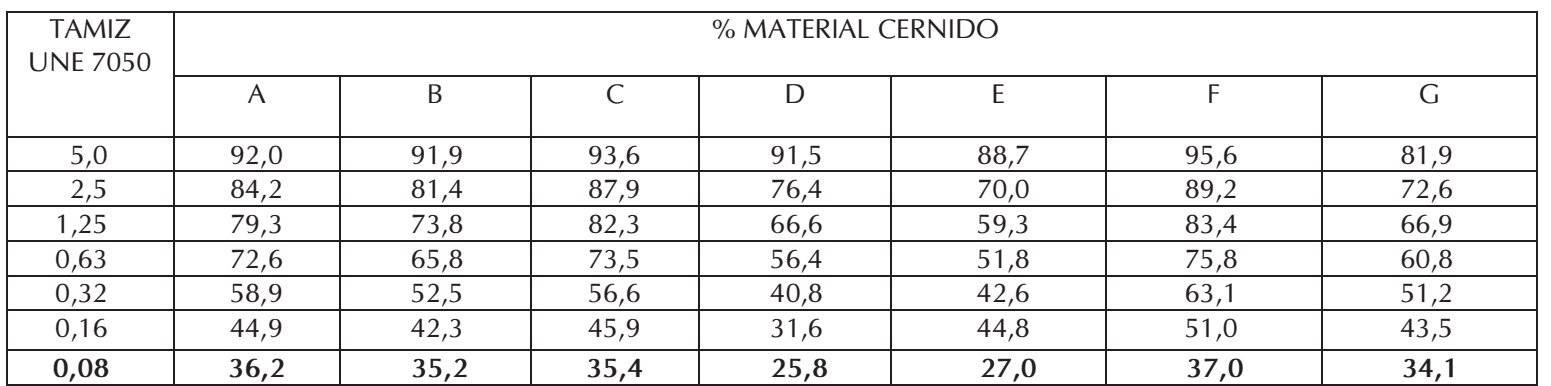

Tabla 4

Valores de resistencia a compresión de los ladrillos

\begin{tabular}{|c|c|c|}
\hline $\begin{array}{c}\text { RESISTENCIA MEDIA } \\
\text { A COMPRESIÓN } \\
\mathrm{N} / \mathrm{mm}^{2}\end{array}$ & DESVIACIÓN TÍPICA & RESISTENCIA CARACTERÍSTICA \\
\hline $26,3 \mathrm{~N} / \mathrm{mm}^{2}$ & $7,6 \mathrm{~N} / \mathrm{mm}^{2}$ & $\mathrm{~N} / \mathrm{mm}^{2}$ \\
\hline
\end{tabular}

rotura donde el valor de tensión admisible a compresión es de 0,21 a 0,4 N/mm² para muros de la misma esbeltez (el valor de la resistencia característica es de 0,84 a 1,6 N/ $\left.\mathrm{mm}^{2}\right)(5)$.

\subsection{Aplicación del Acelerógrafo}

Para medir la rigidez del edificio "in situ" utilizamos un equipo de vibraciones dinámicas con una sensibilidad suficiente para activarse con los movimientos producidos por el tráfico y el viento. El objeto de este ensayo no destructivo el muro estudiado se realizan aproximaciones a partir de la Norma Sísmica vigente NCSE-02.

- Se realiza una estimación del periodo que debería tener el edificio en la actualidad, considerando una altura de 11,30 m y 18,50 $\mathrm{m}$ de longitud. El muro se trata como un elemento ménsula debido a la falta de arriostramiento horizontal con los forjados.

$$
\mathrm{T}=0,06 * \mathrm{H}^{*}((\mathrm{H} /(2 * \mathrm{~L}+\mathrm{H})) / \mathrm{L}) 0,5=0,076 \mathrm{~s}
$$

El módulo de elasticidad que la fábrica de ladrillo debería tener, podría estimarse despejando de la expresión (6):

$$
T=2 \pi / w=2 \pi \sqrt{\frac{m}{E_{d} I}}
$$

Tenemos que $\mathrm{Ed}=42.365 .512 \mathrm{kPa}=4.236$ $\mathrm{N} / \mathrm{mm}^{2}$. El módulo de elasticidad estático es 
aproximadamente el dinámico dividido por 2,5, lo que es lo mismo:

$$
4.236 / 2,5=1694 \mathrm{~N} / \mathrm{mm}^{2}
$$

- El periodo real que hemos determinado con las medidas dinámicas ha sido de $\mathrm{T}=1,25 \mathrm{~s}$. Dividiendo el periodo real por el periodo que debería tener el edificio, vemos que existe una proporción de 16,44. Esto es, la rigidez estructural del edificio es de 1/16,44 veces la que debería tener. El muro de fachada se encuentra por tanto suelto y formado por materiales con módulos de elasticidad bajos.

- Siguiendo las prescripciones del Instituto Eduardo Torroja en su capítulo de "Obras de Fábrica" (7), podemos obtener un valor teórico para el módulo de elasticidad inicial $E_{0}$. Su determinación será función de la resistencia a compresión $\mathrm{s}_{\mathrm{r}}$ mediante la fórmula experimental:

$$
\mathrm{E}_{\mathrm{o}}=\alpha \cdot \sigma_{r}
$$

siendo $\sigma_{\mathrm{r}}$, el valor máximo estimado según análisis de los materiales de $0,4 \mathrm{~N} / \mathrm{mm}^{2}$ y $\alpha$ el coeficiente de deformabilidad de la fábrica (en nuestro caso le correspondería un valor de 2000). El módulo de elasticidad inicial sería por tanto de $800 \mathrm{~N} / \mathrm{mm}^{2}$.

Para el cálculo del módulo de elasticidad correspondiente a otros valores de $\sigma$, podrá determinarse a partir del valor $\mathrm{E}_{\mathrm{o}}$. Para la determinación de las rigideces de los elementos de fábrica, puede tomarse el siguiente valor:

$$
E=0,8 \cdot E_{o}
$$

Siendo $\mathrm{E}=640 \mathrm{~N} / \mathrm{mm}^{2}$.

Tomaremos en este caso como valor estimado del módulo de elasticidad de la fábrica, $\mathrm{E}=$ $700 \mathrm{~N} / \mathrm{mm}^{2}$, algo inferior a la mitad del que le correspondería si los materiales que lo componen estuvieran en buenas condiciones. La aplicación del acelerógrafo permite verificar la vinculación del elemento a las medianeras y forjados y los bajos módulos de elasticidad de sus materiales para la reproducción de un modelo válido para el análisis numérico así como la verificación del estado del muro frente a acciones dinámicas.

\subsection{Análisis numérico. Aplicación del Método de Elementos Finitos a los muros de fábrica de ladrillo portantes}

Para conseguir una comprensión del comportamiento de la fábrica de ladrillo es necesario considerarla como un material elástico, se debe considerar el hecho de que los materiales se deforman cuando reciben una carga, pudiendo así utilizar los conceptos de tensión y deformación. El estudio elástico lineal nos revela estados posibles de equilibrio mediante los mapas de distribución de tensiones máximas de tracción y compresión. Nos permite observar el mecanismo de trabajo de la fábrica sin fisurar, dando una visión general de áreas de concentración de tensiones.

Para nuestro propósito necesitaremos reproducir con la mayor fiabilidad posible las características geométricas y estructurales del edificio, con la finalidad de simular el comportamiento del edificio para una fábrica en el supuesto de no haber llegado a rotura en ningún punto. Nos ayudarán los ensayos previos realizados, así como el conocimiento de los vínculos del muro con el suelo, medianeras y forjados.

- Modelización geométrica de la fábrica. Programa utilizado.

Para poder realizar el análisis propuesto necesitamos un tipo de modelado sólido regular que facilite la generación de una malla de elementos prismáticos. Incluiremos para ello todos los elementos geométricos estructurales necesarios: huecos, pretiles, prescindiendo de los elementos decorativos.La utilización de elementos volumétricos 3-D Ilamados sólidos, dará como resultado un modelo de elementos finitos tridimensional. Con estos elementos se representará el muro en todo su espesor. El nivel de respuesta que obtendremos será estática lineal: desplazamientos, tensiones medias, resultado de un comportamiento elástico del conjunto. En ningún caso se pretende obtener una historia de evolución de fisuras y cargas, para el que este tipo de análisis se ha mostrado insuficiente por distintos especialistas en la materia, debiendo pasar a un análisis no lineal y más complejo para una fábrica tan heterogénea y cuya complejidad nos alejaría del objetivo del método propuesto. A continuación presentamos la modelización del muro de calle Caballerizas (Fig. 9, pág. 26).

- Datos de partida. Hipótesis de carga. Para obtener la respuesta estática lineal, previamente se han introducido los datos de la fábrica de ladrillo en el programa de elementos finitos.

En cada uno de los casos a analizar habrá que aportar datos como:

- Peso específico de la fábrica.

- Espesor del muro.

- Módulo de Elasticidad de la fábrica.

- Vínculos del muro con el suelo y con las medianeras.

- Cargas que afectan al muro. 


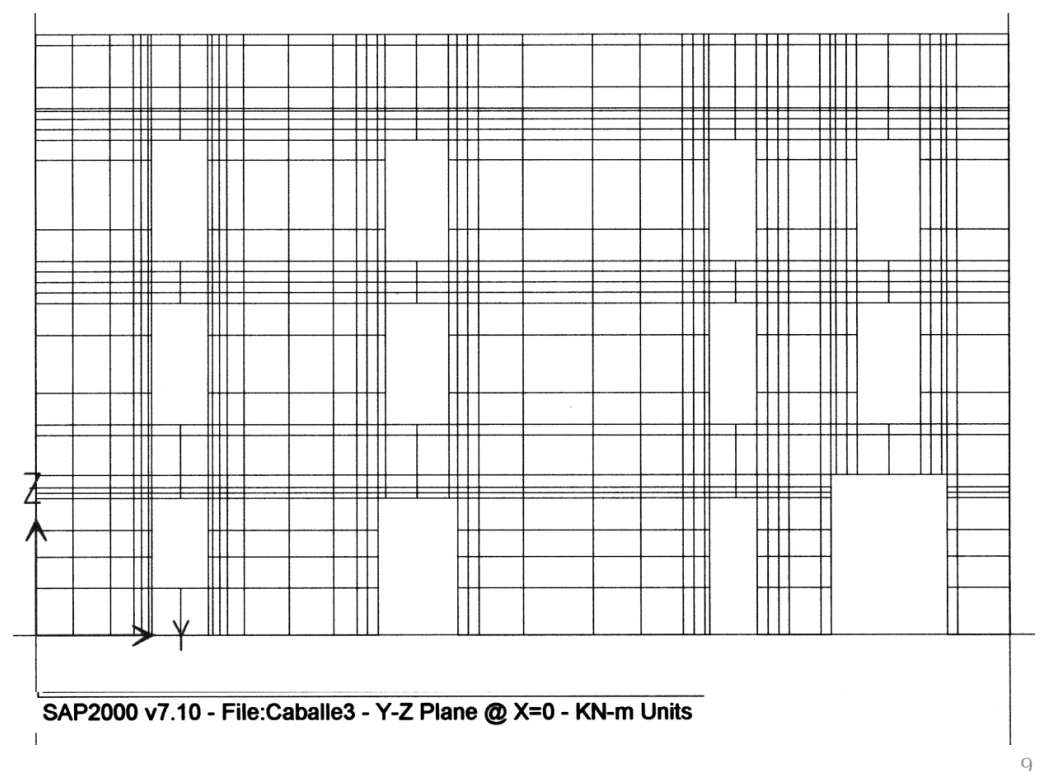

9. Modelización del muro de fachada de Caballerizas 10-12.

Habrá que determinar con anterioridad las hipótesis de carga que queremos analizar. El interés del análisis numérico se centra especialmente en el estado actual del muro, con las cargas que soporta previo a la rehabilitación, pudiendo así, mediante un estudio comparativo del mapa de tensiones obtenido y el plano de lesiones realizado, obtener una tensión de rotura para la fábrica y poder valorar el tipo de intervención más adecuado para conservar la estabilidad del elemento estructural. En general, una reutilización del edificio ante nuevas condiciones de: con cargas, sobrecargas y coeficientes de seguridad en cumplimiento de la Normativa vigente, producirá un incremento de cargas de 0,2 a $0,3 \mathrm{~N} / \mathrm{mm}^{2}$. Es necesario asegurar que el muro podrá soportar este incremento con niveles de seguridad aceptables.

En el caso de Caballerizas 10-12, se han considerado los siguientes datos:

- Peso específico de la fábrica: $1.800 \mathrm{kp} / \mathrm{m}^{3}$. - Espesor de la fábrica: $54 \mathrm{~cm}$.

- Módulo de elasticidad: $700 \mathrm{~N} / \mathrm{mm}^{2}$.

- Se ha considerado el vínculo con el suelo como un empotramiento y con las medianeras una rótula con desplazamiento libre en sentido perpendicular al muro.

- Se considera la hipótesis de carga:

Hip.1 denominada Caballe 2. Peso propio del muro + forjado y solería (condiciones actuales del muro).

\subsection{Ensayos ligeramente destructivos: Gatos planos}

Para la estimación de la tensión de trabajo actual y poder verificar el mapa de tensiones teórico según M.E.F. se han realizado ensayos ligeramente destructivos in situ (gatos planos) en zonas de la fábrica sana cuyo resultado ha sido una tensión de relajación media de $0,15 \mathrm{~N} / \mathrm{mm}^{2}$ (Fig.10).

\subsection{Interpretación de los resultados. Correlación entre gráficos de tensiones y Estado de la Fábrica}

A partir de la introducción de resultados se obtendrán las salidas del programa de cálculo en las que se reflejarán mapas de tensiones principales menores (compresiones) y de tensiones mayores (tracciones) para la hipótesis estudiada (Figs. 11 y 12).

A partir de ellos podemos decir que:

Las máximas compresiones se producen en planta baja a nivel de la mocheta (derecha), de la puerta de mayor anchura, y son del orden de 1,4 N/mm² . La máxima tracción se produce en los cargaderos de los huecos $y$ es del orden de $0,6-0,8 \mathrm{~N} / \mathrm{mm}^{2}$. La interpretación del análisis por el método de los elementos finitos del estado tensional del muro junto con los obtenidos de los ensayos ligeramente destructivos y estudios experimentales nos Ilevan a concluir que:

- La tensión media en las zonas sanas de la fábrica según el gráfico de tensiones mínimas(color amarillo) coincide con el ensayo de gato plano dando tensiones de 0,09 a $0,18 \mathrm{~N} / \mathrm{mm}^{2}$, con lo que verificamos el método de elementos finitos válido para el estado actual de la fábrica sana.

- Hay zonas del muro que actualmente llegan a trabajar a $1,4 \mathrm{~N} / \mathrm{mm}^{2}$ a compresión y a $0,8 \mathrm{~N} / \mathrm{mm}^{2}$ a tracción, cuando hemos determinado una resistencia característica a compresión de la fábrica de $0,85 \mathrm{~N} / \mathrm{mm}^{2}$.

- La desproporción entre lo que resiste la fábrica y las tensiones reales justifica el mal estado de la misma en las zonas de concentración de máximas tensiones (grietas, fisuras, desprendimientos, curvaturas de paños de fábrica completos), lo cual nos permite obtener una tensión de rotura entre 1,0 y $1,2 \mathrm{~N} / \mathrm{mm}^{2}$.

- La aparición de grietas y fisuras han creado zonas sueltas en el muro, rótulas plásticas, falta de continuidad que dificultan la previsión del comportamiento del muro ante una intervención que modifique el estado actual de cargas.

- En este caso el muro está al límite de resistencia. Sólo se garantizaría la seguridad del mismo ante una intervención que respetara los vínculos de éste con los forjados, sin aumentar las cargas y sin poder realizar ninguna modificación en los huecos existentes.

Para garantizar la seguridad del edificio ante las sobrecargas y coeficientes de seguridad actuales sería preciso plantear un refuerzo en la totalidad del muro, la sustitución de din- 


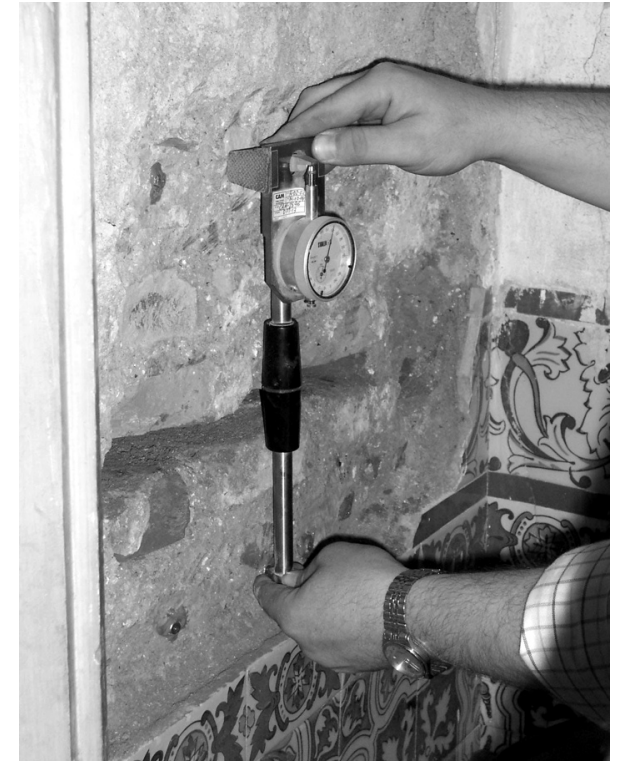

10

teles en los huecos y el refuerzo de jambas. Ante la falta de resistencia frente a acciones horizontales, sería necesario arriostrar el mismo con la vinculación a los elementos perpendiculares.

\section{CONCLUSIONES}

Del desarrollo del método propuesto para el análisis de muros de obras de fábrica podemos concluir:

- El método se desarrolla para un ámbito y una arquitectura determinada con similares características formales, constructivas y de materiales en los elementos estudiados. Es necesario un estudio individualizado de los edificios que incluya información histórica, levantamiento planimétrico, inspección visual del estado de la edificación, y estudio constructivo del elemento.

- Es imprescindible la utilización de técnicas ligeramente destructivas, como la extracción de muestras, para la aproximación a los parámetros resistentes de la fábrica.

- Se ha conseguido mediante un trabajo experimental en laboratorio reproducir la fábrica a partir de los datos anteriores, obteniendo una resistencia a compresión característica proximada de $0,85 \mathrm{~N} / \mathrm{mm}^{2}$, válida para todas las fábricas estudiadas que han coincidido en los parámetros de los materiales que las componen.

- En aquellos casos en que la homogeneidad de la fábrica, la calidad de los materiales y la economía lo aconsejen, se recomienda la utilización de gatos planos para una valoración de la tensión de trabajo de la de la misma.
- La aplicación del acelerógrafo se ha mostrado válida en este tipo de edificios para determinar la rigidez estructural y la respuesta de los mismos ante acciones horizontales, así como las vinculaciones de los muros objeto de estudio para la reproducción del modelo.

- El análisis estático lineal mediante el Método de Elementos Finitos es suficiente para establecer valores comparativos entre el estado tensional de la fábrica suponiéndola sin fisuras en su situación actual de cargas y los datos obtenidos con los distintos ensayos, corroborándose en todos los casos las secciones de agotamiento del material a compresión con las zonas que han superado la resistencia a compresión determinada por el método descrito, pudiendo incluso aproximarnos a la tensión de rotura de la fábrica estudiada.
10. Ensayo de gato plano. Determinación de tensiones.

11. Diagramas de tensiones mínimas (compresiones). Hipótesis 1, Caballerizas $10-12$

12. Diagramas de tensiones mínimas (tracciones). Hipótesis 1, Caballerizas 10-12.

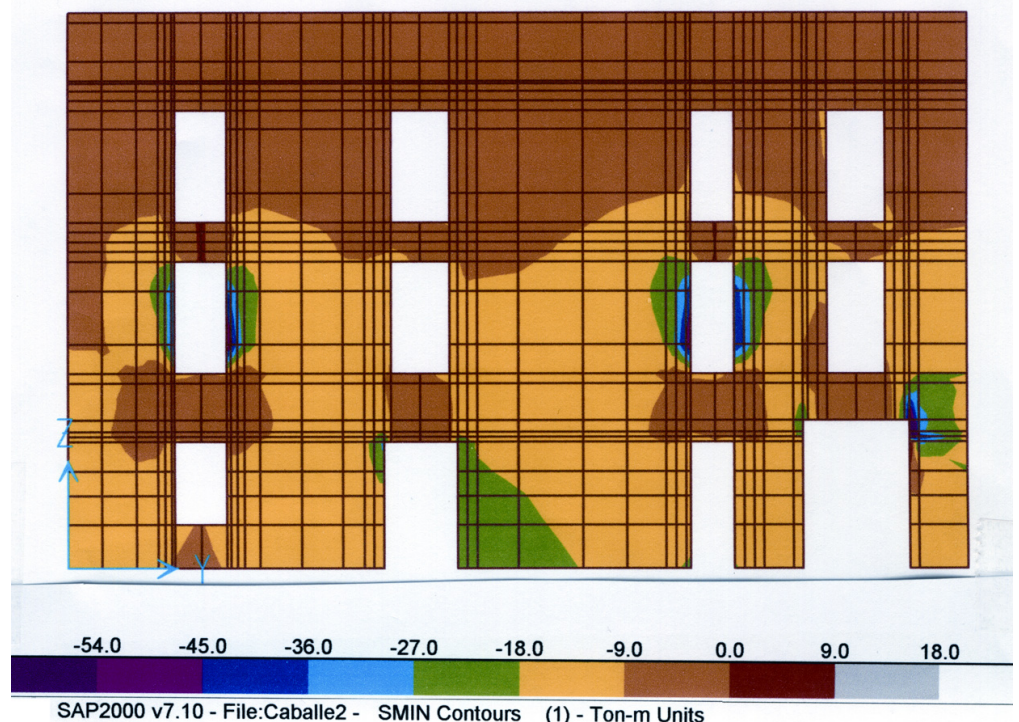

SAP2000 v7.10 - File:Caballe2 - SMIN Contours (1) - Ton-m Units

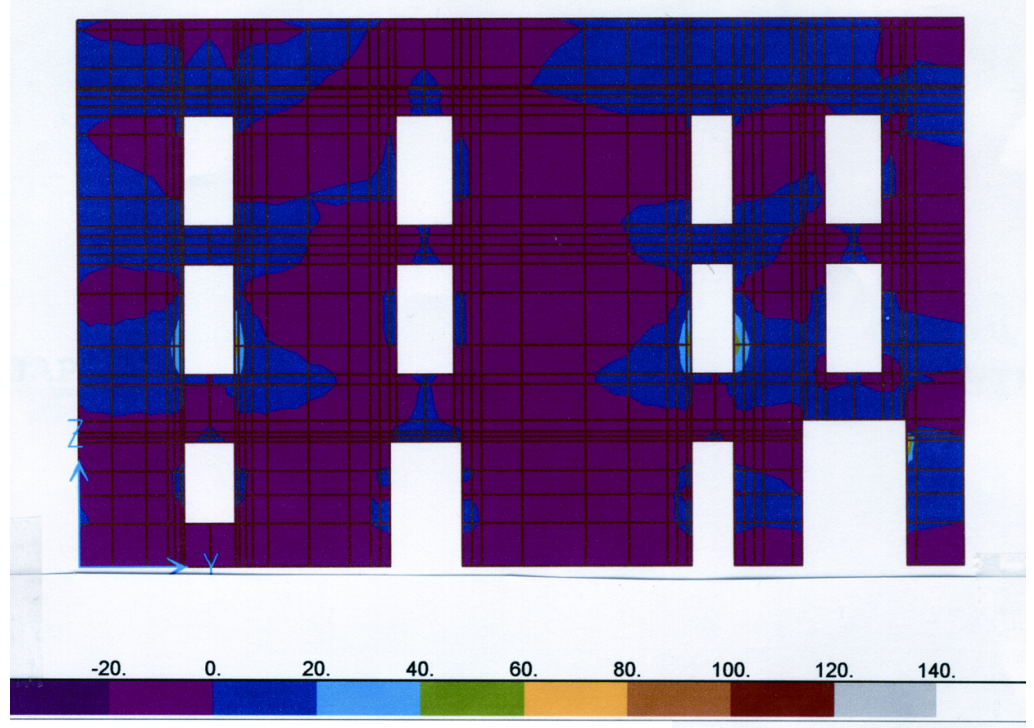

SAP2000 v7.10 - File:Caballe2 - SMAX Contours (1) - Ton-m Units 


\section{BIBLIOGRAFÍA}

(1) Oñate, E.: "Cálculo de estructuras por el método de elementos finitos". CIMNE, 1998.

Como, M.; lanniruberto, U.; Imbimbo, M.: "A rigid plastic model of the under-excavation technique applied to stabilise leaning towers". Actas de Historical Constructions. Possibilities of numerical and experimental techniques. Guimaraes 2001.

Lourenco P.B.: "Análisis of historical constructions: from trust-lines to avances simulations". Actas de Historical Constructions. Possibilities of numerical and experimental techniques. Guimaraes 2001.

(2) Sierra Delgado, J.R: "La casa en Sevilla 1976-1996". Ed. Fundación El Monte y Electa Sevilla 1996.

(3) NBE FL-90. Norma Básica de la Edificación. Muros resistentes de fábrica de ladrillo. Ministerio de Obras Públicas y Urbanismo, 1991.

(4) Pérez Gálvez, F.: "Las obras de fábrica en la Arquitectura Doméstica sevillana de los siglos XVIII y XIX. Características constructivas y parámetros resistentes". Tesis Doctoral, Universidad de Sevilla, 2004.

(5) Soto Pardo, M.: "Reforma de edificios antiguos. El rasgado de huecos en muros de carga". Informes de la Construcción, Vol. 37, n³ 374, 1985.

(6) Jaramillo Morilla, A.: "Método probabilístico de estimación de las acciones sísmicas". Tesis doctoral. E.T.S.A. de Sevilla 1983. Capítulo V.

(7) Instituto Eduardo Torroja: P.I.E.T. 70. Obras de fábrica. Madrid, 1971. 